\title{
Measuring and Correcting Response Heaping Arising From the Use of Prototypes
}

\section{JAY BEAMAN, ${ }^{1}$ JERRY J. VASKE, ${ }^{1}$ JENNIFER I. SCHMIDT, ${ }^{2}$ AND TZUNG-CHENG HUAN ${ }^{3}$}

${ }^{1}$ Human Dimensions of Natural Resources, Colorado State University, Fort Collins, Colorado, USA

${ }^{2}$ Institute for Social and Economic Research (ISER), University of Alaska Anchorage, Anchorage, Alaska, USA

${ }^{3}$ College of Management, National Chiayi University, Chiayi, Taiwan

\begin{abstract}
Imprecision in respondent recall can cause response heaping in frequency data for particular values (e.g., 5, 10, 15). In human dimensions research, heaping can occur for variables such as days of participation (e.g., hunting, fishing), animals/fish harvested, or money spent on licenses. Distributions with heaps can bias population estimates because the means and totals can be inflated or deflated. Because bias can result in poor management decisions, determining if the bias is large enough to matter is important. This note introduces the logic and flow of a deheaping program that estimates bias in means and totals when people use approximate responses (i.e., prototypes). The program can make estimates even when spikes occur due to bag limits. The program is available online, and smooths heaps at multiples of 5 (numbers ending in 5 and 0 ) and 7 (e.g., 7, 14, 21), and produces standard deviations in estimates.
\end{abstract}

Keywords response heaping, deheaping, prototype use, estimate bias

\section{Introduction}

Surveys are commonly used for obtaining data on hunter and angler behavior. When asked to recall a frequency (e.g., days of participation, number of trips) or quantity (e.g., trip expenditures, game harvested), respondents may give approximate answers by using prototypes (Vaske \& Beaman, 2006). A prototype is a single number characterizing a range of values (Huttenlocher, Hedges, \& Bradburn, 1990). If asked about frequency of fishing, for example, a person may recall fishing somewhere between 8 and 12 times per month. The prototype used in a survey response might be 10. Prototypes are suggested by response heaps (i.e., peaks in response frequency functions) (Figure 1). Heaps appear because some responses occur more often than would be expected by chance (Chase \& Harada, 1984; Hultsman, Hultsman, \& Black, 1989). If prototype responses bias results,

Address correspondence to Tzung-Cheng Huan, Dean, College of Management, National Chiayi University, 580 Hsin-Ming Road, Chiayi, 60054 Taiwan. E-mail: tchuan@mail.ncyu.edu.tw 


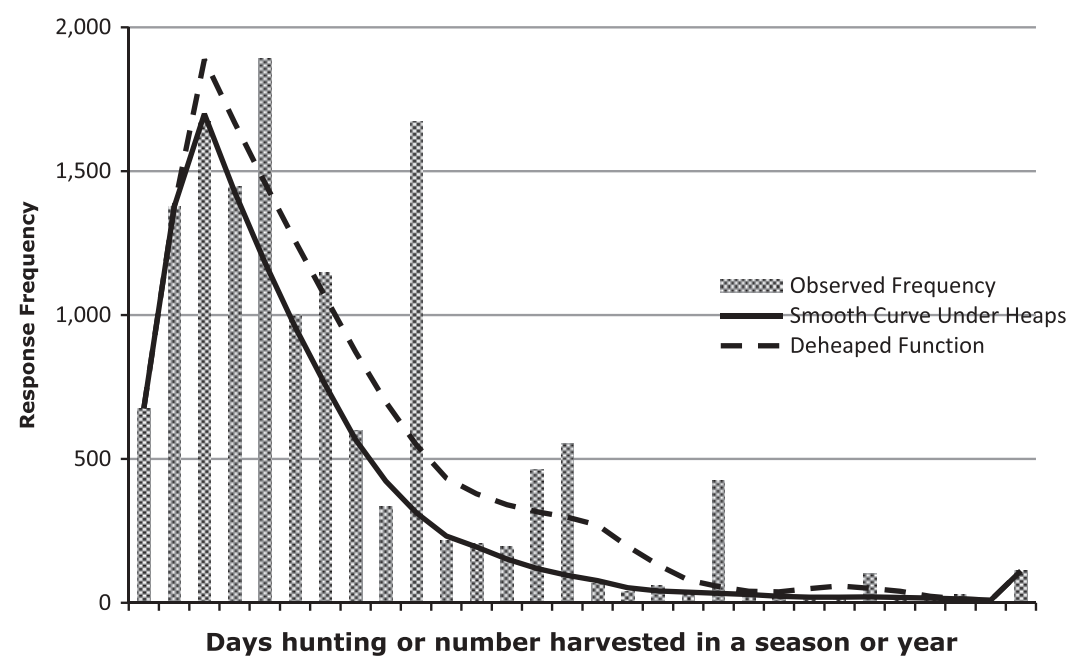

Figure 1. Frequency function appropriate for deheaping.

the utility of estimates for planning and management is compromised. Some researchers (e.g., Camarda, Eilers, \& Gampe, 2008) have proposed mathematical models for smoothing response heaping, but mathematical smoothing may not reflect how people behave. This research note describes a response smoothing (i.e., deheaping) program that is based on how respondents recall results in heaped responses from the use of prototypes.

\section{Past Research}

When answering questions about behavior, respondents may use a variety of cognitive processes such as digit preference, formula-based multipliers, and prototypes (Burton \& Blair, 1991; Nadeau \& Niemi, 1995). Early human dimensions research defined digit preference in terms of an individual's preference for numbers ending in 0 or 5 (Chase \& Harda, 1984; Hultsman et al., 1989; Tarrant \& Manfredo, 1993). Other research, however, has shown that response heaps at 4 and 8 are possible when the variable "hours of participation during a day" is examined (Rodgers, Brown, \& Duncan, 1993). If the variable of interest is "duration of a trip" (in days), responses of 7, 14, 30, and 60 tend to be overused (Huttenlocher et al., 1990) because they correspond to weeks or months. For "respondent age," individuals tend to overuse numbers ending in 9 (e.g., 29, 39, 49) and under-report those ending in 0 (e.g., 30, 40, 50; Bailey \& Makannah, 1993). The "digit preference" phenomenon, therefore, should be referred to as "number preference," as it does not generally relate to numbers ending in certain digits, but rather to numbers that a person has a disposition to use or avoid (Beaman, Vaske, \& Grenier, 1998; Vaske \& Beaman, 2006; Vaske, Beaman, Manfredo, Covey, \& Knox, 1996).

If the number of events (e.g., hunting trips, annual harvest) to be recalled is low (e.g., $1,2)$, traditional memory models assume that people use episode enumeration (Sudman \& Bradburn, 1974; Sudman, Bradburn, \& Schwarz, 1996). As the frequency of participation in a behavior and/or quantity to be estimated increases, episodic enumeration can yield to estimation heuristics where frequency is estimated by recalling sample episodes (Tversky \& Kahneman, 1974). More salient episodes tend to be recalled more frequently, resulting in overestimation (Vaske, Huan, \& Beaman, 2003). When events are numerous, similar, and 
occur regularly, respondents may use formula-based multipliers (Burton \& Blair, 1991). For this cognitive process, individuals employ a frequency rule and apply it to the requested time frame. For example, an individual may recall going fishing once a week and when asked how many times he or she fished in the last three months, the response might equal 12 (i.e., once a week $* 4$ weeks per month $* 3$ months $=12$ ).

The recall process may also involve the use of prototypes, which are single numbers characterizing a range of values (Huttenlocher et al., 1990). For example, if an individual recalls hunting somewhere between 18 and 22 times in a season, the prototype response might be 20 . Another individual, however, may reply 20 feeling the appropriate response is between 15 and 25 . Responses in a heap, therefore, may result from the use of more than one prototype (Beaman, et al., 1998).

Prototype responses are not accurate, but they are also not necessarily biased. Problems arise because the word "bias" has two meanings. First, bias can refer to answers that are intentionally misleading. For example, respondents might lie about how often they use illegal drugs or their willingness to pay increased access fees (Krosnick, 1999). The former is often biased downward, whereas the latter often has a positive bias (Whittaker, Vaske, Donnelly, \& DeRuiter, 1998). Second, bias can result from ways that researchers calculate estimates (e.g., means, totals) when responses include prototype responses. Although prototypes reflect how the human mind works (Burton \& Blair, 1991), bias occurs because researchers make the error of treating prototypes as accurate and precise responses (Beaman et al., 1998).

Prototypes ending in 0 or 5 are not necessarily prototype or inaccurate responses. Given that $20 \%$ of all numbers end in 0 or 5 , about one-fifth of all responses (e.g., harvest, days) will end in 0 or 5 when no preference is given to multiples of 5 . A response of 5 days hunting may be perfectly accurate. The problem is determining for a given response (e.g., 10), how many responses are the result of using prototypes. If $25 \%$ of respondents provide answers ending in 0 or 5 , all $25 \%$ might implicitly be classified as using prototypes. Alternatively, one could argue that only $5 \%(25 \%-20 \%=5 \%)$ of respondents used prototypes. Given this logic, the question becomes, which is closer to the actual percent using prototypes?

Beaman, Vaske, Donnelly, and Manfredo (1997) developed an unbiased aggregate measure of prototypes ending in 0 or 5 . Computation of this aggregate measure involved estimating the total number of respondents who could have used the prototype. In subsequent work (Beaman, Vaske, \& Miller, 2005a, 2005b), a formula was derived for estimating the proportion of responses in a heap.

Existing research has assumed that respondent prototypes are symmetric around a heaping value (Beaman et al., 1998). Given that heaps are more widely separated as the magnitude of responses increases, larger prototype responses may be asymmetric (Vaske $\&$ Beaman, 2006). In addition, the use of broad prototypes increases with the magnitude of responses. For responses greater than 35, for example, a reported harvest of 50 may have a prototype range of 30 and 75 . On the other hand, a prototype range for 15 might be 13 to 17 . For 15 , the only prototype may be $15 \pm 2$. For 20 , however, some people may round to multiples of 5, while others might round to multiples of 10 .

Models for correcting data containing prototype heaps have been proposed (e.g., Beaman et al., 1998). The 1998 model, as well as the current model, are based on a series of assumptions:

Assumption 1: A cognitive recall process occurs when a respondent reports a number.

Assumption 2: When respondents are uncertain of the exact number to give, approximate or prototype values may be given. 
Assumption 3: Prototype answers are unbiased in the sense that a prototype response is equally likely for any value in the range of the prototype (i.e., for $15 \pm 2$ whether the correct response is $13,14,15,16$, or 17 does not influence the probability of responding by 15$)$.

Assumption 4: Respondents use context-sensitive prototypes (e.g., 0 or 5 prototypes may be used when reporting harvest; 7,14 , or 21 prototypes when reporting days of participation).

Assumption 5: Respondents use a limited number of prototypes (e.g., $5 \pm 2,10 \pm 2$, $10 \pm 5,15 \pm 2$; or $7 \pm 3,14 \pm 3$, or $21 \pm 3$ ).

Assumption 6: As the magnitude of responses increases, the heaping pattern changes. For example, for responses $<35$, prototypes are numbers ending in 0 and 5 are used; for larger values, prototype use is dominated by responses ending in 0 .

Assumption 7: Using prototypes results in heaps on some numbers (i.e., peaks in a frequency distribution).

Assumption 8: Distributions with heaped responses can be approximated by a smooth frequency function.

\section{Deheaping Program}

Efforts at modeling response heaping have evolved over the past two decades. A preliminary version of a model was outlined in Vaske et al. (1996), and important ideas were clarified in Beaman et al. (1997, 1998). Refinements to the 1998 approach are discussed on the website (www.auctorresearch.net/deheap). A deheaping program can be downloaded from the website.

The deheaping program approximates how prototypes are used. In other words, the program estimates a "deheaped" function approximating the frequency function that would have been observed if heaping was not present (e.g., see "Deheaped Function" in Figure 1). When a deheaped function has been estimated, bias (e.g., in mean days of hunting) due to heaping is determined by comparing an estimate based on the deheaped function with the estimate obtained using actual responses. Standard deviations can also be requested. These are determined by introducing random variation into observations (see Step 7 below) and making estimates for the randomized responses. From 5 to 99 randomizations can be used. Steps that occur in estimation include:

Step 1. Identify where prototype response heaps occur in the data. Consider the frequency function shown in Figure 1. Peaks in the data are evident for 0 and 5 heaping (i.e., 5, 10, 15, 20) and multiples of 7 (i.e., 7, 14). The current program can also estimate spikes resulting from daily bag limit constraints (e.g., a daily harvest limit of 4 ducks).

Step 2. Determine the number of responses in each heap using regression to establish where heaps begin. Regression determines the "smooth curve under the heaps" (Figure 1). For example, based on total responses minus the value at the smooth curve, there are about 1,900 responses in the heap at 5, and slightly less than 1,200 in the heap at 7 (Figure 1).

Step 3. For each prototype heap, the program identifies prototypes that are likely being used for a given response. For heaps at 10 and 20, the program specifies prototypes of $10 \pm 2$ and $10 \pm 5$ and $20 \pm 2$ and $20 \pm 5$ contributing to heaps (i.e., the heaps at 10 and 20 are taken to be sharing responses from two prototypes). 
Step 4. Use the "smooth curve under the heaps" (Figure 1) to allocate responses in heaps back to approximately where they came from. This curve provides an initial estimated shape for the deheaped function being estimated (i.e., $\mathrm{d}_{0}(\mathrm{x})$ )). For heaps at multiples of 10 (e.g., the heap at $\mathrm{K}^{*} 10$ where $\mathrm{K}$ is an integer), distributing back involves recognizing that a proportion of the responses in the heap comes from the use of prototype $\mathrm{K}^{*} 10 \pm 2$ and must be distributed back to that range, whereas the rest come from $\mathrm{K}^{*} 10 \pm 5$ and must be distributed back to that broader range. For details on heap sharing and estimation of proportions see "PROGRAM 3: A Deheaping Program for Estimating Best Heap Sharing Ratios for 10-heaps" at www.auctorresearch.net/deheap.

Step 5. The deheaping program iteratively moves from the initial estimate, $\mathrm{d}_{0}(\mathrm{x})$, to an improve allocation, $\mathrm{d}_{1}(\mathrm{x})$, by using $\mathrm{d}_{0}(\mathrm{x})$ to determine how responses in heaps are distributed "back." The function $\mathrm{d}_{1}(\mathrm{x})$ is then used for arriving at $\mathrm{d}_{2}(\mathrm{x})$. The goal is responses distributed consistent with the value of the smooth function. Repeated testing with different frequencies has shown that five iterations produce estimates that do not change by $25 \%$ of their standard deviation (i.e., continued iteration does not change estimates significantly). Therefore, the program uses five iterations to obtain estimates (e.g., estimation using $\mathrm{d}_{4}(\mathrm{x})$ yields the estimate of the $\mathrm{d}_{5}(\mathrm{x})$ ("Deheaped Function" in Figure 1).

Step 6. To obtain standard deviations in estimates (e.g., standard deviation in bias in mean days hunted or percent of respondents in heaps), observed frequencies are randomly simulated between 5 and 99 times. The default is five, but researchers can select a large number of simulations. Greater accuracy can be achieved by basing standard deviations on 10 or 20 randomizations with estimates, but processing time takes longer. The results from each simulation are saved and used to calculate the standard deviation in the estimate.

Step 7. Standard deviations are computed, stored and output based on having $n$ observations of estimates.

Step 8. Results are presented in a graphic like Figure 1.

\section{Running the Deheaping Program}

Download the deheaping program from www.auctorresearch.net/deheap going to "Quick start for using the deheaping program." The zip file contains a SAS program, documentation for running, sample data files, and example output. Documentation provides detailed screen-by-screen guidance on running the deheaping program. Example text from one of the output files is shown below. Data for this example were obtained from a 1997 Alaskan moose hunting harvest report study (see Schmidt \& Chapin, 2014, for details). Bias was estimated at $2.8 \%$ (i.e., the presence of heaps that resulted in overestimation) with a standard deviation in bias of about $.12 \%$ (i.e., $2.8 \%$ has a $S D$ of .12\%). From a practical perspective, total days hunting of 138,213 was based on 19,169 harvest report permits, whereas there were 134,245 deheaped total days of hunting (i.e., sum of days for the 19,169 permits). In other words, the raw data overestimated participation by approximately 4,000 days.

-Iteration 5 (Final) for dayshunt

Mean \& total bias \%:

Estimated \% of respondent heapers: $26.0 \%$

Observed total dayshunt: 
Deheaped total dayshunt:

Total respondents:

Observed mean days hunting:

Deheaped mean days hunting:

For 5 randomizations,

Mean bias for mean and total dayshunt:

Mean $\%$ of respondents in heap:
134,245

19,169

7.2

7.0

$\begin{array}{ll}2.75 \% & \text { STD }=0.118 \% \\ 25.84 \% & \text { STD }=0.749 \%\end{array}$

\section{Discussion}

This note provided an introduction to a deheaping program that researchers can use to examine the effect of response heaping on estimates resulting from the use of prototypes. Prototypes are suggested by peaks in response frequency functions. Such heaps occur because some responses occur more often than would be expected by chance. If prototype responses bias results when treated as accurate responses, the utility of estimates for planning and management is compromised. Other researchers (e.g., Camarda et al., 2008) have used sophisticated mathematical modeling for smoothing data containing response heaping. Such models, however, may not mirror the cognitive processes that individuals use to recall a frequency (e.g., days of participation, number of trips) or quantity (e.g., trip expenditures, game harvested). The deheaping program here makes estimates based on previous human dimensions research showing the thought patterns behind responses being in heaps when prototypes are used. The program can be used to determine the percent of respondents in heaps or to estimate the percent of bias in estimates produced by the use of prototypes.

This article focused on heaping for multiples of 5 and of 7 because past research suggests that multiples of these values occur most commonly. Future research might consider estimating response heaps for other values. Our focus on multiples of 5 and 7, however, did introduce estimation issues (e.g., 5 and 10-heapers in heaps at multiples of 10, and 10-heapers in overlapping intervals). Current research on these issues is available at www. auctorresearch.net/deheap. Overall, given the potential negative consequences associated with making management decisions using data containing response heaps, researchers are encouraged to use the programs to identify the impact of heaping on estimates.

\section{References}

Bailey, M., \& Makannah, T. J. (1993). Patterns of digit preference and avoidance in the age statistics of some recent African censuses: 1970-1986. Journal of Official Statistics, 9, 705-715.

Beaman, J., Vaske, J. J., Donnelly, M. P., \& Manfredo, M. J. (1997). Individual versus aggregate measures of digit preference. Human Dimensions of Wildlife, 2, 71-80.

Beaman, J., Vaske, J. J., \& Grenier, M. (1998). A prototype model for estimating and correcting bias in digit preference/number preference. Tourism Analysis, 2, 77-90.

Beaman, J., Vaske, J. J., \& Miller, C. A. (2005a). Cognitive processes in hunters' recall of participation and harvest estimates. Journal of Wildlife Management, 69, 967-975.

Beaman, J., Vaske, J. J., \& Miller, C. A. (2005b). Hunting activity record-cards and the accuracy of survey estimates. Human Dimensions of Wildlife, 10, 285-292.

Burton, S., \& Blair, E. (1991). Task conditions, response formulation processes and response accuracy for behavioral frequency questions in surveys. Public Opinion Quarterly, 55, 50-79. 
Camarda, C. G., Eilers, P. H. C., \& Gampe, J. (2008). Modelling general patterns of digit preference. Statistical Modelling, 8, 385-401.

Chase, D. R., \& Harada, M. (1984). Response error in self-reported recreation participation. Journal of Leisure Research, 16, 322-329.

Hultsman, W. Z., Hultsman, J. T., \& Black, D. R. (1989). "Response peaks" as a component of measurement error: Assessment implications for self-reported data in leisure research. Journal of Leisure Research, 21, 310-315.

Huttenlocher, J., Hedges, L. V., \& Bradburn, N. M. (1990). Reports of elapsed time: Bounding and rounding processes in estimation. Journal of Experimental Psychology: Learning, Memory and Cognition, 16, 196-213.

Krosnick, J. A. (1999). Survey research. Annual Review of Psychology, 50, 537-567.

Nadeau, R., \& Niemi, R. G. (1995). Educated guesses: The process of answering factual knowledge questions in surveys. Public Opinion Quarterly, 59, 323-346.

Rodgers, W. L., Brown, C., \& Duncan, G. J. (1993). Errors in survey reports of earnings, hours worked, and hourly wages. Journal of the American Statistical Association, 88, 1208-1218.

Schmidt, J. I., \& Chapin, F. S. (2014). Relationship of community characteristics to harvest reporting: Comparative study of household surveys and harvest tickets in Alaska. Human Dimensions of Wildlife, 19, 334-346.

Sudman, S., \& Bradburn, N. M. (1974). Response effects in surveys: A review and synthesis. Chicago, IL: Aldine.

Sudman, S., Bradburn, N. M., \& Schwarz, N. (1996). Thinking about answers: The application of cognitive processes to survey methodology. San Francisco, CA: Jossey-Bass.

Tarrant, M. A., \& Manfredo, M. J. (1993). Digit preference, recall bias, and nonresponse bias in self reports of angling participation. Leisure Sciences, 15, 231-238.

Tversky, A., \& Kahneman, D. (1974). Judgment under uncertainty: Heuristics and biases. Science, $185,1124-1131$.

Vaske, J., \& Beaman, J. (2006). Lessons learned in detecting and correcting response heaping: Conceptual, methodological and empirical observations. Human Dimensions of Wildlife, 11, 285-296.

Vaske, J. J., Beaman, J., Manfredo, M. J., Covey, D., \& Knox, R. (1996). Response strategy, recall frame, and digit preference in self-reports of angling participation. Human Dimensions of Wildlife, $1,54-68$.

Vaske, J. J., Huan, T. C., \& Beaman, J. G. (2003). The use of multiples in anglers' recall of participation and harvest estimates: Some results and implications. Leisure Sciences, 25, 399-410.

Whittaker, D., Vaske, J. J., Donnelly, M. P., \& DeRuiter, D. S. (1998). Mail versus telephone surveys: Potential biases in expenditure and willingness to pay data. Journal of Park and Recreation Administration, 16(3), 15-30. 